\title{
Group infall of substructures on to a Milky Way-like dark halo
}

\author{
Yang-Shyang Li and Amina Helmi \\ Kapteyn Astronomical Institute, University of Groningen, \\ P.O. Box 800, 9700 AV Groningen, the Netherlands \\ email: ysleigh@astro.rug.nl; ahelmi@astro.rug.nl
}

\begin{abstract}
We report the discovery that substructures/subhaloes of a galaxy-size halo tend to fall in together in groups in cosmological simulations, something that may explain the oddity of the MW satellite distribution. The original clustering at the time of infall is still discernible in the angular momenta of the subhaloes even for events which took place up to eight Gyrs ago, $z \sim 1$. This phenomenon appears to be rather common since at least $1 / 3$ of the present-day subhaloes have fallen in groups in our simulations. Hence, this may well explain the LyndenBell \& Lynden-Bell ghostly streams. We have also found that the probability of building up a flattened distribution similar to the MW satellites is as high as $\sim 80 \%$ if the MW satellites were from only one group and $\sim 20 \%$ when five groups are involved. Therefore, we conclude that the 'peculiar' distribution of satellites around the MW can be expected with the CDM structure formation theory. This non-random assignment of satellites to subhaloes implies an environmental dependence on whether these low-mass objects are able to form stars, possibly related to the nature of reionization in the early Universe.
\end{abstract}

Keywords. Methods: $N$-body simulations, Galaxy: formation, galaxies: dwarf, galaxies: kinematics and dynamics.

\section{Introduction}

The discrepancy in numbers between the substructures/subhaloes resolved in a galaxysize cold dark matter (CDM) halo and the satellites around the Milky Way (MW) has been a long standing issue for the 'concordance' CDM structure formation theory. It implies a non-trivial mapping between the luminous satellites and the dark matter subhaloes at the (sub) galactic scales (Zentner et al. 2005; Libeskind et al. 2007) through the astrophysical processes with baryons or the theory need major modification at a fundamental level (see e.g. Kamionkowski \& Liddle 2000).

In the past ten years new attention has been drawn to the dynamical properties of the MW satellites. Starting with Lynden-Bell \& Lynden-Bell (1995), the existence of ghostly streams of satellites (dwarf galaxies and globular clusters) was postulated. These objects would share similar energies and angular momenta producing a strong alignment along great circles on the sky. Recently, the anisotropic distribution of satellites around the MW has been argued to be a problem for the CDM theory (Kroupa, Theis \& Boily, 2005; Metz, Kroupa \& Jerjen, 2007). The MW has approximately 20 satellites forming a disk-like structure while the simulated dark matter subhaloes usually distribute almost isotropically.

Here we report our findings of subhaloes falling in groups in dark matter simulations and its application to explain the oddities of the dynamical properties of MW satellites, namely, the Lynden-Bell \& Lynden-Bell ghostly steams and the great MW satellites disk. Research in the past has showed that clusters of galaxies are built of galaxies coming in groups (Knebe et al. 2004), but it was not clear whether a similar picture also applies at 
the (sub)galactic scale. We refer readers to a more detailed description of our analyses and discussion of the group infall and its link to the environment in Li \& Helmi (2008).

\section{Substructures in a galaxy-size dark halo}

\subsection{The N-body Simulations}

To study the dynamical properties of dark matter subhaloes, we have analysed the GAnew series of high resolution simulations of a MW-like halo in a full cosmological context $\left(\Omega_{0}=0.3, \Omega_{\Lambda}=0.7, \mathrm{H}_{0}=100 h \mathrm{~km} \mathrm{~s}^{-1} \mathrm{Mpc}^{-1}\right.$ and $\left.h=0.7\right)$. The simulations were carried out with GADGET-2 (Springel 2005) and a more detailed description on the simulation itself are reported in Stoehr (2006). In the highest mass resolution simulation (GA3new), about $10^{7}$ particles within the virial radius resolve the MW-like halo at $z=0$. These simulations are abundant with self-bound substructures $(\sim 4,000$ in GA3new $)$ and the starting redshifts of the simulations are as high as $z=37.6$, therefore rendering the simulations ideal for studies of subhalo populations and dynamics.

\subsection{Group infall of dark matter subhaloes}

The degree of clustering is quantified by computing the two-point 'angular correlation function', $\omega(\alpha)$, of the present-day angular momentum of the subhaloes.

$$
\omega(\alpha)=\frac{N\left(\alpha_{i j}<\alpha\right)_{\text {simulation }}}{N\left(\alpha_{i j}<\alpha\right)_{\text {isotropic }}}-1
$$

The angle $\alpha$ is the relative orientation of the angular momenta of any two subhaloes, i.e. $\cos \alpha_{i j}=\mathbf{L}_{i} \cdot \mathbf{L}_{j} /\left(\left|\mathbf{L}_{i}\right|\left|\mathbf{L}_{j}\right|\right)$. Therefore the correlation function measures the number of pairs, $N$, with $\alpha_{i j}<\alpha$ seen in the simulations compared to what is expected from an isotropic distribution with the same number of objects. An excess of pairs at small angular separations indicate small scale clustering in the present-day angular momentum. This clustering in the angular momentum space is still discernible even for some groups accreted about eight Gyrs ago $(z \sim 1)$.

We now focus on the characteristics of groups accreted at various epochs. To identify groups we link pairs of infalling haloes whose angular momentum orientations are separated less than ten degrees, i.e., $\alpha<10^{\circ}$, and with relative distances $d<40 \mathrm{kpc}$ at the time of accretion. We found that this combination of $\alpha$ and $d$ values results in a robust set of groups, maximising their extent while minimising the number of spurious links.

We then follow the orbits of the groups identified from redshift $z \sim 4.2$ until present time. Fig. 1 shows the trajectories of some of the richest groups of subhaloes, which were accreted 2.43, 1.65 and 0.84 Gyrs ago respectively. Each dot represents the position of a subhalo colour coded from high-redshift (dark) to the present (light). The blue symbols correspond to the present-day positions while those at the time of accretion are shown in red. Fig. 1 clearly shows that groups of subhaloes follow nearly coherent orbits even long before being accreted.

\section{Mass function of groups}

We have also looked at the mass distribution of groups of subhaloes at the time of accretion. The mass function is dominated by low mass groups and can be fitted with a power-law, $d N / d \log M \propto M^{n}$ with $n \sim-0.5 \pm 0.15$. Note that this slope is slightly shallower compared to the mass function of the full subhalo population in a galaxy and a galaxy cluster size dark halo where $n=-0.7--0.9$ (e.g., Gao et al. 2004). At the limit of our simulations, we also examined the mass spectra within individual groups. 


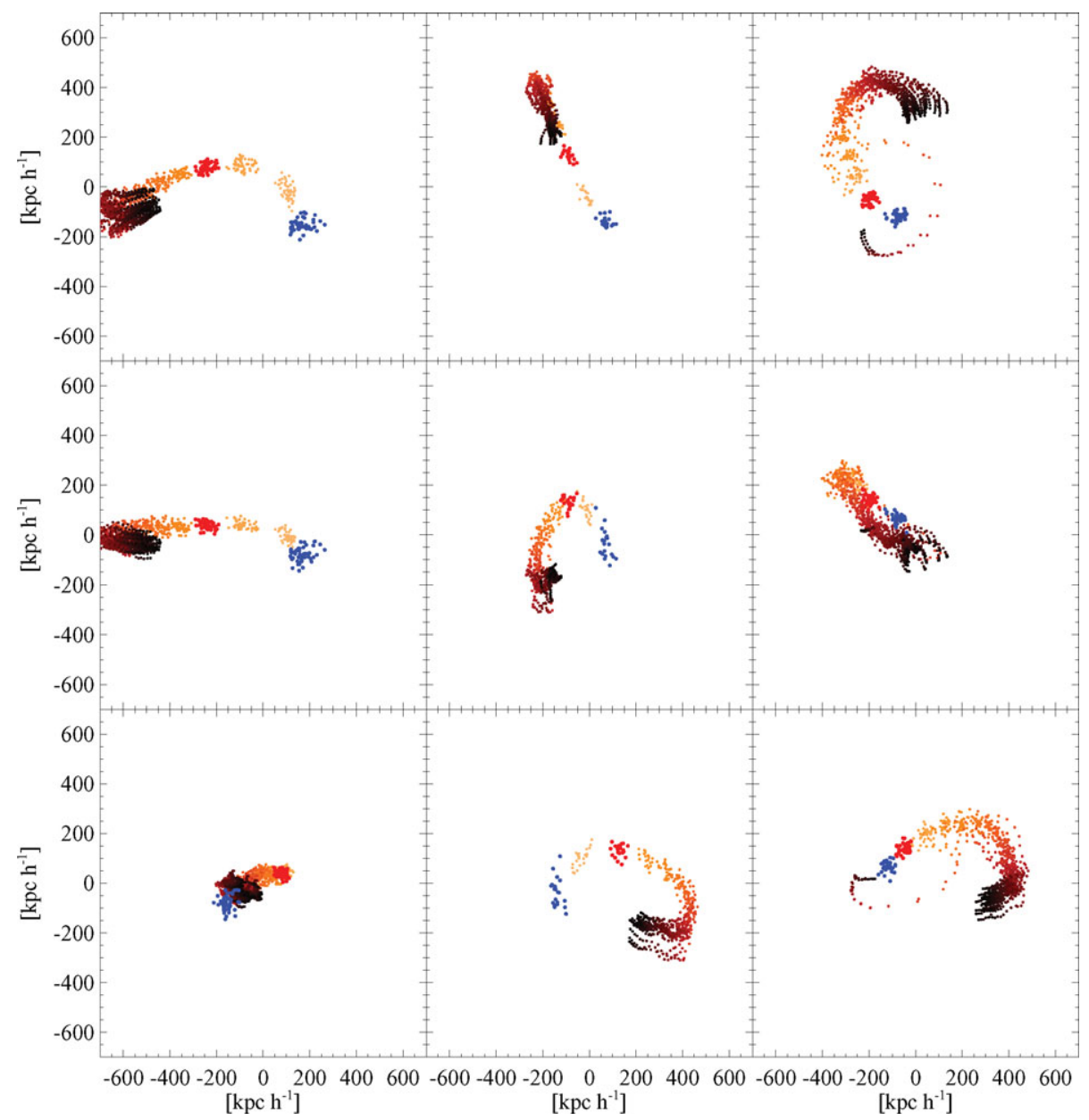

Figure 1. Three examples of trajectories of groups of subhaloes accreted at different epochs in the GA3new simulation reference frame projected onto different planes. These are some of the most abundant groups ever accreted. The colour gradients indicate the arrow of time, from dark at high redshift to light grey at the present. The positions at accretion and present time are highlighted in red and blue respectively.

The data suggests the mass distribution of subhaloes also follows a power-law trend in a group, although the signal is much noisier.

\section{On the dynamical peculiarities of the MW satellites}

\subsection{Lynden-Bell \& Lynden-Bell ghostly streams}

Fig. 1 shows the group members following similar orbits and retaining the coherence for several Gyr. The clustering of subhaloes in the space is most prominent when they accrete onto the MW-like halo. But later on, the spatial coherence of groups will be destroyed due to the gravitational interactions with the host and the group members will spread along the orbit. We note that this characteristic of groups would naturally lead to structures sharing common orbital planes, analogous to Lynden-Bell \& Lynden-Bell ghostly streams. Thus group infall provides an explanation clearly different from the 
disruption of a massive progenitor (Lynden-Bell \& Lynden-Bell, 1995) or the tidal-origin scenario (Kroupa 1997).

\subsection{Great disk of Milky Way satellites}

As stated in the Introduction, the distribution of the MW satellites is not isotropic. Kroupa, Theis \& Boily (2005) have shown that the satellites form a highly flattened 'Great Satellites Plane' whose rms distance to this plane is $10-30 \mathrm{kpc}$. These authors conclude that this anisotropic structure is inconsistent with that of the subhaloes seen in the CDM simulations. Here we reconsider this issue with our high resolution CDM simulations. In our analyses, we focus on the eleven 'classical' MW satellites since it is not clear whether the newly discovered SDSS satellites confirm the flattened disk-like structure due to the limited sky coverage.

The great satellite disk of the classical MW satellites has an orientation of $72.8 \pm 0.7^{\circ}$ with respect to the Galactic plane (see Fig. 2 left panel). Here we follow the definition by Zentner et al. (2005) to describe the flatness of a satellites disk as $\Delta=D_{r m s} / R_{m e d}$ where $D_{r m s}$ is the rms distance to the best-fitted plane, and $R_{m e d}$ is the median distance of objects. For the MW satellites, the flatness of the great disk is $\Delta=0.23 \pm 0.01$ with $D_{\text {rms }} \sim 18.5$ and $R_{\text {med }} \sim 80 \mathrm{kpc}$.

Of the 3,246 subhaloes within $300 \mathrm{kpc}$ from the centre of the MW-like halo that have survived until the present day, 898 subhaloes fell in as part of a group. This means that about $1 / 3$ of the surviving subhaloes have joined the MW-like halo in a group-infall fashion. 321 different groups have contributed to the present-day population of subhaloes, of which the earliest two were accreted at $z=3.05$. From now on, surviving subhaloes identified to be part of a group are referred to as 'grouped', while those which are not are termed 'field' subhaloes.

The idea now is to test whether groups of subhaloes would form a disk distribution similar to the great MW satellite disk. We have performed two simple tests as follows:

(a) Consider $N_{\text {sub }}$ 'grouped' subhaloes accreted from only one group and $11-N_{\text {sub }}$ from the field.

(b) Consider only the 'grouped' subhaloes originated in a few groups.

We generate $10^{5}$ sets of eleven subhaloes from the present-day population which satisfy either of the above mentioned conditions and compute the flatness of the best-fit planes. The fraction of disks is then the number of sets with flatness as low as that of the great satellites disk, i.e., $N\left(\Delta \leqslant \Delta_{\mathrm{GSD}}\right)$, normalised by the total number of realisations. The result shows that when considering case (a), the disk fraction increases from $4.5 \%$ to $73 \%$ as the number of selected subhaloes $N_{\text {sub }}$ increases from 2 to 11 . In comparison, 11 randomly-selected subhaloes (within $300 \mathrm{kpc}$ ) gives rise to flattened configurations $\sim 2.2 \%$ of the time. This shows that if the Milky Way satellites fell in together, it would not be very surprising that they would be in a planar configuration at the present-day.

When considering only subhaloes originating in groups (case b), the fraction of disklike configurations obtained in this way can be as high as $\sim 40 \%$ when the subhaloes come from only two groups, and of course reaches $73 \%$ when they come from just one group. It is important to note that though the disk fraction decreases to $\sim 20 \%$ when selecting from 5 different groups, it is still much higher than if one selects 11 subhaloes randomly. On the right panel of Fig. 2, we show an example of the distribution of three groups of subhaloes with 3,4 , and 4 group members respectively projected along their best-fitted plane. This distribution has $\Delta \sim 0.17$.

Given the large fraction of flattened configurations found in our simulations, we conclude that the spatial distribution of the 11 Milky Way satellites can be reproduced 


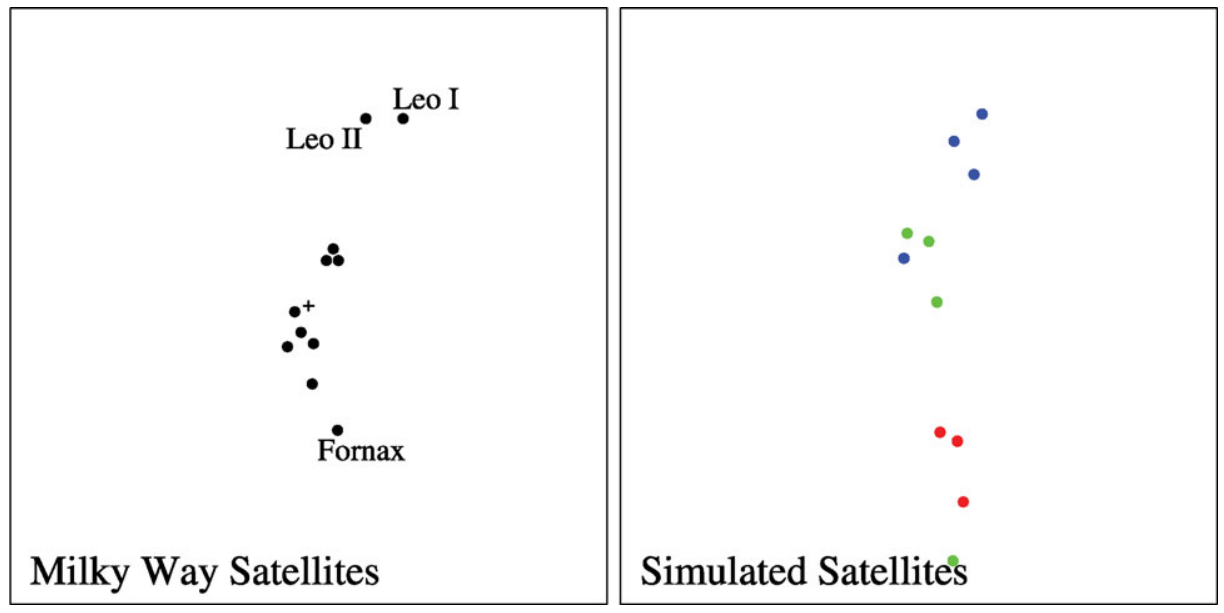

Figure 2. Left: Illustration of the distribution of the eleven classical Milky Way satellites where the cross sign denotes the Galactic centre. The Galactic disk would be horizontal in this orientation. Right: An example of the distribution of satellites in our simulations which can be traced back to come from three different groups as marked by different colours.

within $\Lambda \mathrm{CDM}$. The requirement is that these satellites fell onto the Galactic halo in groups.

\section{Conclusions and Implications}

We have revisited the issue of the peculiar distribution and properties of the MW satellites and their link to the dark matter subhaloes. In particular, we have focused on the infall of substructures on to a Milky-Way like dark matter halo in a $\Lambda$ CDM cosmogony utilising a series of high-resolution dark-matter simulations. We have found evidence of group infall on to the MW-like halo, which may explain the ghostly streams proposed by Lynden-Bell \& Lynden-Bell (1995).

We have also explored how this planar configuration may be obtained as a result of the infall of satellites in groups. The observed correlation in the angular momentum orientation of subhaloes naturally gives rise to disk-like configurations. For example, we find that if all subhaloes are accreted from just one group, a disk-like distribution is essentially unavoidable ( $\sim 80 \%$ probability), while for accretion from just two groups, the likelihood of obtaining a distribution as planar as observed is $40 \%$. Therefore the disky configuration of satellites is consistent with CDM if most satellites have their origin in a few groups. Note that in our studies, we do not need to invoke the baryon-related physics to account for the dynamical properties of the MW satellites. Thus both the 'ghostly streams' and the 'planar configuration' are manifestations of the same phenomenon: the hierarchical growth of structure down to the smallest galactic scales.

One of the possible implications of the reality of the ghostly streams is that its member galaxies formed and evolved in a similar environment before falling into the MW potential. This would have implications on the (oldest) stellar populations of these objects, such as for example, sharing a common metallicity floor (Helmi et al. 2006). On the other hand, this implies that there should be groups that have failed to host any luminous satellites. This would hint at a strong dependence on environment of the ability of a subhalo to retain gas (Scannapieco et al. 2001), or be shielded from re-ionization by nearby sources (Mashchenko, Carignan \& Bouchard 2004; Weinmann et al. 2007). 
Recent proper motion measurements of the Large and Small Magellanic clouds by Kallivayalil, van der Marel \& Alcock (2006), as well as the simulations by Bekki \& Chiba (2005) suggest that these systems may have become bound to each other only recently. This would be fairly plausible in the context of our results. The Clouds may well have been part of a recently accreted group and it may not even be necessary for them to ever have been a binary system.

\section{Acknowledgements}

YSL thanks the organising committee for giving the opportunity to contribute this talk and the travel grant supported by IAU. This work has been supported by a VIDI grant from the Netherlands Foundation for Scientific Research (NWO).

\section{References}

Bekki, K. \& Chiba, M. 2005, MNRAS, 356, 680

Gao, L., White, S. D. M., Jenkins, A., Stoehr, F., \& Springel, V. 2004, MNRAS, 355, 819

Helmi, A. et al. 2006, ApJ, 651, L121

Kallivayalil, N., van der Marel, R. P., \& Alcock C. 2006, ApJ, 652, 1213

Kamionkowski, M. \& Liddle, A. R. 2000, Phys. Rev. Lett., 84, 4525

Knebe, A., Gill, S. P. D., Gibson, B. K., Lewis, G. F., Ibata, R. A., \& Dopita, M. A. 2004, ApJ, 603,7

Kroupa, P., Theis, C., \& Boily, M. 2005, A\&A, 431, 517

Kroupa, P. 1997, New Astron., 2, 139

Li, Y.-S. \& Helmi A. 2008, MNRAS, 385, 1365

Libeskind, N. I., Cole, S., Frenk, C. S., Okamoto, T., \& Jenkins, A. 2007, MNRAS, 374, 16

Lynden-Bell, D. \& Lynden-Bell, R. M. 1995, MNRAS, 275, 429

Metz, M., Kroupa, P., \& Jerjen, H. 2007, MNRAS, 374, 1125

Mashchenko, S., Carignan, C., \& Bouchard, A. 2004, MNRAS, 352, 168

Scannapieco, E., Thacker, R. J., \& Davis, M. 2001, ApJ, 557, 605

Stoehr, F., White, S. D. M., Tormen, G., \& Springel, V. 2002, MNRAS, 335, L84

Springel V. 2005, MNRAS, 364, 1105

Stoehr, F. 2006, MNRAS, 365, 147

Weinmann, S. M., Macció, A. V., Iliev, I. T., Mellema, G., \& Moore, B. 2007, MNRAS, 381, 367

Zentner, A. R., Kravtsov, A. V., Gnedin, O. Y., \& Klypin, A. A. 2005, ApJ, 629, 219 